\title{
AZUL OU ROSA? A SEGREGAÇÃO DE GÊNERO NO ENSINO SUPERIOR BRASILEIRO, 2002-2016
}

Tayná Mendes I, (D) Luiza Houzel II

D Bruna Milanski III, (iD Carolina Medeiros ${ }^{\text {IV }}$

(D) Flávia Eduarda Rochav, (D) Pedro Elgalyvi

(D) Vivian de Almeida VII, (iD Flavio Carvalhaes viI

I, II, III, IV, V, VI, VII, VIII Universidade Federal do Rio de Janeiro (UFRJ), Núcleo Interdisciplinar de Estudos sobre a Desigualdade (NIED-IFCS-UFRJ), Rio de Janeiro (RJ), Brasil; mendesttayna@gmail.com

\section{Resumo}

O artigo examina como estudantes dos sexos masculino e feminino estão alocados em diferentes grupos de curso do ensino superior brasileiro em 2002 e em 2016. Foram utilizados os microdados da Pesquisa Nacional por Amostra de Domicílios 2014 e do Censo do Ensino Superior 2002 e 2016. Os resultados indicam que a conclusão do ensino superior é desproporcionalmente feminina, padrão que se intensificou na última coorte avaliada. Por outro lado, existem diferenças significativas indicando que mulheres estão sobrerrepresentadas em grupos de curso com menores retornos no mercado de trabalho. O padrão de segregação de gênero das escolhas educacionais é estável ao longo do tempo. No entanto, observou-se que isso não se deve a um comportamento estático da segregação de gênero nos grupos de curso, mas sim à compensação de cursos que diminuem e aumentam a segregação.

\section{RELAÇÕES DE GÊNERO • ENSINO SUPERIOR・ESTRATIFICAÇÃO SOCIAL}

\section{BLUE OR PINK? GENDER SEGREGATION IN BRAZILIAN HIGHER EDUCATION,} 2002-2016

\footnotetext{
Abstract

The article examines how male and female students were allocated to different groups of Brazilian higher education courses in 2002 and 2016. Microdata from the 2014 Pesquisa Nacional por Amostra de Domicílios [National Household Sample Survey] and the 2002 and 2016 Censo do Ensino Superior [Higher Education Census] were used. The results indicate that the number of female students that complete high school is disproportionately higher, and that it increased in the more recent born cohort. On the other hand, there are significant differences showing that women are over-represented in fields of study with lower returns in the labor market. The gender segregation pattern of educational choices is stable over time. However, it was observed that this is not due to a static behavior of gender segregation in the fields of study, but to the compensation of undergraduate courses that decrease and increase segregation.
} 


\section{¿AZUL O ROSA? SEGREGACIÓN DE GÉNERO EN LA EDUCACIÓN SUPERIOR BRASILEÑA, 2002-2016}

\section{Resumen}

El artículo examina cómo se asignanlos estudiantes masculinos y femeninos a diferentes grupos de cursos de educación superior de Brasil en 2002 y 2016. Fueron utilizados los microdatos de la Pesquisa Nacional por Amostra de Domicílios [Encuesta Nacional por Muestreo de Hogares] de 2014 y del Censo do Ensino Superior [Censo de Educación Superior] 2002 y 2016. Los resultados indican que la finalización de la educación superior es desproporcionadamente femenina, lo que aumentó en la última generación evaluada. Por otro lado, existen diferencias significativas que indican que las mujeres están sobrerrepresentadas en los grupos de cursos con menores rendimientos en el mercado laboral. El patrón de segregación de género de las opciones educativas es estable a lo largo del tiempo. Sin embargo, se observó que esto no se debe a un comportamiento estático de segregación de género en los grupos de cursos, sino más bien a la compensación de cursos que disminuyen y aumentan la segregación.

RELACIONES DE GÉNERO • ENSEÑANZA SUPERIOR • ESTRATIFICACIÓN SOCIAL

\section{BLEU OU ROSE ? SÉGRÉGATION DE GENRE DANS L'ENSEIGNEMENT SUPÉRIEUR AU BRÉSIL, 2002-2016}

\section{Résumé}

L'article examine comment les étudiants hommes et femmes sont répartis entre les différentes filières de l'enseignement supérieur brésilien en 2002 et 2016. Les microdonnées utilisées proviennent de la Pesquisa Nacional por Amostra de Domicílios [Enquête Nationale auprès des Ménages] de 2014 et du Censo do Ensino Superior [Recensement de l'Enseignement Supérieur] pour 2002 et 2016. Les résultats indiquent que les femmes sont disproportionnellement représentées parmi ceux qui achèvent leurs études supérieures, leur nombre ayant augmenté au cours de la dernière génération étudiée. Toutefois, il existe des différences significatives montrant que les femmes sont surreprésentées dans les filières les moins rentables sur le marché du travail. Le modèle de ségrégation entre les sexes concernant les choix éducatifs semble être stable dans le temps. Ceci n'est cependant pas dû au comportement statique de la ségrégation de genre dans les filières, mais à un phénomène de compensation entre les cours qui réduisent et augmentent la ségrégation.

RELATIONS DE GENRE • ENSEIGNEMENT UNIVERSITAIRE • STRATIFICATION SOCIALE 
LONGEVIDADE DE CARREIRAS ESCOLARES É MAIOR PARA MULHERES DO QUE HOMENS AO redor do mundo (DiPrete \& Buchmann, 2013) e no Brasil (Beltrão \& Alves, 2009). Apesar disso, mulheres continuam tendo resultados socioeconômicos - salários, estabilidade no emprego - sistematicamente piores do que homens, independentemente do país analisado (Bobbitt-Zeher, 2007; para o Brasil, ver Galvão, 2015). A literatura aponta que a razão para o hiato salarial de gênero no mercado de trabalho está associada a vários processos relacionados à família e ao trabalho (Itaboraí, 2016). Em relação ao último domínio, um componente importante é a segregação ocupacional. Os salários de homens e mulheres não são iguais, em parte, porque eles e elas trabalham em diferentes ocupações, sendo que, na média, as carreiras com sobrerrepresentação masculina têm remuneração maior.

Entre os indivíduos mais escolarizados, esse tipo de hiato se repete (Galvão, 2015). Assim, é importante entender tanto a segregação ocupacional para homens e mulheres que atingem um ponto de alta escolarização - o ensino superior - como o que a alimenta, ou seja, compreender a segregação das escolhas educacionais antes da entrada no mercado de trabalho. Este artigo é uma contribuição nesse sentido.

\section{Revisão da literatura}

A partir da década de 1960, houve uma tendênciaglobal de aumento do número de estudantes matriculados no nível superior de ensino, resultado de um processo de intensa expansão da educação terciária (Schofer \& Meyer, 2005). Uma característica advinda desse processo foi a maior participação de mulheres em níveis educacionais mais elevados, o que provocou uma reversão da tendência da desigualdade de gênero no acesso ao ensino superior em vários países. As mulheres, que em diversos contextos nacionais tinham trajetórias educacionais mais curtas do que os homens, passaram a concluir níveis de ensino mais avançados ao redor do mundo (Charles \& Bradley, 2002).

A literatura especializada no tema, em vários países (Buchmann et al., 2008) e no Brasil (Beltrão \& Alves, 2009), indica que a média de anos de estudo de mulheres é maior do que a dos homens. Nos níveis educacionais mais elevados - ensino superior e pós-graduação -, as mulheres são maioria. No entanto, a maior longevidade das trajetórias educacionais femininas convive com outro resultado identificado em muitos países: a segregação de gênero das escolhas educacionais. Pesquisadores atentos aos padrões de presença feminina e masculina nos níveis de ensino mais avançados - principalmente o superior identificam uma sistemática diferença de escolhas educacionais entre estudantes de ambos os sexos.

Tal fato, em si, não é um problema, mas as mulheres costumam escolher cursos de ensino superior que acarretam sua sobrerrepresentação em profissões com menor retorno financeiro no mercado de trabalho, como pedagogia, serviço social e enfermagem, conforme apontam pesquisas no Brasil (Carvalhaes \& Ribeiro, 2019). Os homens, por sua vez, operam sistematicamente no sentido oposto, estando sobrerrepresentados em profissões de áreas técnicas, como engenharias e computação (Charles \& Bradley, 2002, 2009). Entender a relação entre gênero e escolhas educacionais, além de ser uma base interessante por si só para uma investigação sociológica, contribui para compreender um dos processos que alimentam a segregação ocupacional, uma parte não trivial do hiato salarial por sexo no mercado de trabalho (Charles \& Grusky, 2004; Galvão, 2015).

A literatura geralmente recorre a conceitos como estereótipos de gênero e essencialismo de 
gênero para explicar esse tipo de padrão empírico. Esses dois processos seriam a base de promoção de vieses no conjunto de habilidades, preferências e crenças que são transmitidas intergeracionalmente e sustentam a permanência de escolhas diferentes entre homens e mulheres. Os mesmos seriam incorporados tanto na própria socialização familiar como na socialização escolar dos indivíduos, já a partir da socialização básica (Brito, 2006; Toledo \& Carvalho, 2018). Essas socializações, agregadas, produzem um padrão de segregação de gênero a partir de escolhas individuais de estudantes dos sexos masculino e feminino. $\mathrm{O}$ conceito de essencialismo de gênero propõe que uma escolha educacional pode ser vista como uma instância em que indivíduos agem de acordo com expectativas societais de comportamentos. No Ocidente, os padrões mais tipicamente encontrados associam tarefas analíticas e manuais a signos masculinos, e tarefas artísticas e de cuidado a signos femininos (Thébaud \& Charles, 2018).

As referências culturais são a base para entender os padrões empíricos tipicamente encontrados: a sobrerrepresentação de mulheres em carreiras humanistas e de cuidado e de homens em carreiras científicas e técnicas (Barone, 2011). Remetendo aos conceitos de estereótipos e essencialismo de gênero, sociólogas apontam que estudantes estão enraizados em um ambiente saturado de informações sobre o que constitui um comportamento "adequado" e estereotipado do ponto de vista do gênero, bem como constantemente reforçado por pais, professores e outros membros de suas redes de sociabilidade.

Segundo Senkevics e Carvalho (2015), há uma cobrança maior sobre meninas para a realização dos afazeres domésticos, que acaba incentivando o desenvolvimento de posturas como organização, responsabilidade e disciplina, o que beneficia no desempenho escolar. Além disso, a instituição escolar adquire para meninos e meninas significados diferentes daquele que a casa e a rua assumem para ambos. Enquanto para meninos o ambiente de casa e a rua apresentam mais possibilidades de lazer e socialização, com maiores permissões para sair e circular, para meninas a casa é um espaço de obrigações domésticas e a rua é um ambiente mais restrito; logo, a escola assume, para meninas, um lugar de lazer e sociabilidade, o que as incentiva a não evadirem e participarem de atividades extracurriculares. Nesse processo, poderiam ser localizadas as origens da performance de gênero, por meio de sua ligação com as concepções normativas dominantes de feminilidade e masculinidade (Thébaud \& Charles, 2018). Essa é a base conceitual da explicação de um padrão de segregação das escolhas de gênero no ensino superior, que opõe cursos de conteúdo humanista (preferidos por mulheres) àqueles de conteúdo científico (preferidos pelos homens).

A origem de outro tipo de segregação - no polo cuidado-técnico - também é remetida a estereótipos, mas de outra categoria. Aqui, os argumentos culturalistas destacam como os estereótipos de gênero estão relacionados à propensão "natural" de as mulheres se ligarem às atividades de cuidado (Cech, 2013). Mas o que significa esse termo, "cuidado"? A literatura sobre relações de gênero e trabalho (England, 2005; Hirata, 2010; Hirata \& Guimarães, 2012) aponta que ocupações ligadas ao cuidado têm duas características: a interação face a face com clientes e tarefas associadas diretamente a seu bem-estar e/ou desenvolvimento pessoal. Logo, as carreiras referentes ao "cuidado" seriam aquelas que, literal ou simbolicamente, envolveriam o componente de interação e a prestação de serviços ligados ao bem-estar. $\mathrm{O}$ argumento que relaciona escolhas educacionais a cursos da área de cuidado destaca que os estereótipos de gênero operam via processos normativos e de socialização que direcionam mulheres e homens para escolhas típicas e segundo gênero em suas carreiras. Implicitamente, há a sugestão de que as preferências masculinas e femininas diferem em relação à família (mais importante para elas) e ao trabalho (mais importante para eles). O resultado agregado é a segregação de gênero em carreiras ligadas aos cursos com conteúdo curricular referente ao cuidado em oposição aos conteúdos técnicos (Weeden et al., 2020).

Diversos estudos testaram empiricamente a importância dos estereótipos de gênero na escolha de cursos de ensino superior entre estudantes dos sexos masculino e feminino, que estavam fazendo a transição do ensino médio para o ensino terciário. Os resultados são contundentes na identificação dos padrões mencionados anteriormente em países variados, como Alemanha (Occhsenfeld, 2016), Itália (Barone \& Assireli, 2020), Estados Unidos (England \& Li, 2006) e Brasil, como será visto a seguir. 
Além de propor os fundamentos conceituais que explicariam o padrão da desigualdade de gênero, a literatura sociológica também reflete sobre sua resiliência temporal, tendo em vista que pesquisas com desenhos que comparam os padróes das escolhas educacionais de homens e mulheres ao longo do tempo apontam que a segregação de gênero é resiliente, ou seja, há pouca variação em sua força quando diferentes coortes (gerações) são comparadas. Potencialmente, esses padrões poderiam ser apenas uma reminiscência do passado e estar diminuindo para, eventualmente, se diluir. Não é o que se observa em países como Itália, Espanha, Noruega, Áustria e Alemanha (Barone, 2011). Como, então, explicar a estabilidade temporal dos padrões de segregação? Charles e Bradley (2002) sugerem que os estereótipos de gênero são facilmente combinados com o conceito de individualidade. Assim, ainda que haja nas sociedades contemporâneas uma rejeição ao sexo como justificativa adscrita para a estruturação de oportunidades, este também pode ser acionado como uma forma de afirmação da diferença "natural" de talento ou preferências, porque não é visto pelo próprio ator como algo discriminatório. Esse processo é remetido quando os pesquisadores se dedicam a responder por que há pouca variação temporal nos padrões de segregação observados.

\section{Pesquisas sobre relações de gênero e escolhas de curso de ensino superior no Brasil}

Segundo Alves (2003), a reversão do hiato de gênero na educação foi a maior conquista das mulheres brasileiras no SéculoXX. Beltrão e Alves (2009), mobilizando dados de diferentes Censos Demográficos, documentam a partir de quando esse padrão pode ser observado. Seguindo o grupo etário de 10-14 anos, os autores constatam que o fenômeno pode ser identificado desde o censo de 1960: os homens dessa faixa etária tinham 1,5 ano de escolaridade em 1960, 2,13 em 1970, 2,24 em 1980, 2,57 em 1991 e 3,6 em 2000; para as mulheres, esses valores correspondem a 1,6, 2,30, 2,52, 2,93 e 3,89 anos, respectivamente (Beltrão \& Alves, 2009, p. 140). Assim, quando se caminha dos grupos etários mais velhos para os mais novos, as mulheres vão reduzindo a diferença em relação à maior escolaridade masculina, sendo que nos grupos mais jovens a diferença se reverte em favor delas. $\mathrm{O}$ hiato de gênero e sua reversão são identificados entre mulheres nascidas após 1950. Em relação às mulheres nascidas entre 1951 e 1955 e identificadas no Censo de 1960, havia 5\% de vantagem em relação aos homens. Nos inquéritos populacionais posteriores a diferença em favor das mulheres aumentou ainda mais.

Segundo Ribeiro e Schlegel (2015), a proporção de mulheres diplomadas cresceu entre 1960 e 2010, "mas os modelos revelam que a diminuição das desigualdades entre gêneros se deu apenas entre 1960 e 1980 e de 2000 a 2010, mantendo-se constante entre 1980 e 2000” (2015, p. 14). Os dados apontam para algumas tendências que ocorrem simultaneamente ao longo do período analisado. Apesar de ter havido uma considerável equalização entre homens e mulheres na conclusão de diversas carreiras universitárias nesse período de crescimento, ainda existem aquelas que são mais femininas e outras que, a despeito da reversão do hiato de gênero entre as pessoas que acessaram o ensino superior entre 1960 e 2010, continuam predominantemente masculinas. Os autores medem a sobre ou sub-representação de homens e mulheres por meio de razões de chances calculadas a partir de modelos log-lineares. A estatística é sensível a qualquer grau de maior ou menor presença dos grupos nas tabelas utilizadas para cálculo, sendo que os autores não destacam a intensidade da segregação, mas sim sua presença ou ausência identificada a partir do modelo ajustado. Assim, carreiras como Letras, Biologia, Belas Artes, Geografia e História seguiram predominantemente femininas no período, enquanto Direito, Veterinária, Medicina, Economia, Religião, Agronomia, Engenharias e Militar de nível superior são predominantemente masculinas. Os autores apontam que nem tudo se manteve estável no período: carreiras como Arquitetura, Odontologia e Matemática-Física-Estatística-Química passaram a ser femininas, essas últimas em decorrência da alta presença das licenciaturas em sua composição. 
Ricoldie Artes (2016) mobilizam dados dos Censos Demográficos e do Censo do Ensino Superior, ambos de 2010, para comparar a paridade de gênero em diversos cursos do ensino superior brasileiro. $\mathrm{O}$ estudo apresenta um foco em 20 áreas de formação, que representam 83,3\% dos estudantes matriculados no ensino superior em 2010 e 78,7\% dos graduados, segundo os dados do Instituto Brasileiro de Geografia e Estatística (IBGE). As autoras apontam que, independentemente da base de dados utilizada, cinco áreas de estudo, que concentram $50 \%$ de matrículas ou formados, têm diferenças bem definidas por sexo. Além disso, observam-se a intensa sobrerrepresentação masculina nas áreas de Engenharia e a feminina em Ciências da Educação.

Carvalhaes e Ribeiro (2019), empregando dados de ingressantes do Exame Nacional de Desempenho dos Estudantes (Enade) de 2007, 2008 e 2009, mostram que o sistema de ensino superior brasileiro segue a tendência apontada pela literatura internacional de presença e intensidade da segregação de gênero nos cursos superiores do país. Segundo os autores, os dados indicam que há maior probabilidade de mulheres estarem em cursos relacionados à docência e aos cuidados de saúde. Cursos como Pedagogia, Nutrição, Fonoaudiologia, Serviço Social, Enfermagem, Psicologia, Biomedicina, Farmácia, Biologia e Odontologia, por exemplo, têm sobrerrepresentação feminina significativa. Ao mesmo tempo, mulheres estão sub-representadas em cursos como Engenharia, Agronomia, Ciências Econômicas, Estatística, Física, Matemática, História, Filosofia, Direito, Educação Física, Administração, Tecnológicos e Medicina.

Esses estudos brasileiros trazem contribuições importantes no sentido de mobilizar dados que apontam para a presença da desigualdade horizontal de gênero no país e seu padrão de estruturação ao longo do tempo. No entanto, eles contêm alguns limites. A utilização de censos demográficos em alguns estudos é limitada do ponto de vista temporal. Na melhor das hipóteses, é possível identificar pessoas já formadas até a última pesquisa censitária brasileira realizada em 2010. Carvalhaes e Ribeiro (2019) empregam dados mais recentes, de ingressantes no ensino superior brasileiro entre 2007 e 2009 , porém seus exercícios não fazem nenhuma comparação temporal, impossibilitando tirar qualquer tipo de conclusão sobre o comportamento da segregação de gênero ao longo do tempo.

Outra ordem de crítica diz respeito à mensuração da segregação de gênero. A comparação temporal de qualquer tipo de estatística tem de ser capaz de isolar como o crescimento geral de um sistema pode afetar os padrões de associação entre seus componentes. Tecnicamente, as medidas de segregação utilizadas têm de ser insensíveis à expansão geral do sistema, pois medidas que não são insensíveis às margens representam um problema amplamente conhecido na literatura sobre mobilidade social (Ribeiro, 2007) e segregação ocupacional por gênero (Charles \& Grusky, 1995, 2004). Os trabalhos que não podem ser criticados sob esse prisma são limitados por não retratarem a segregação de gênero no ensino superior em períodos mais recentes (Ribeiro \& Schlegel, 2015) ou por não terem explicitamente a comparação temporal em seu desenho de pesquisa (Carvalhaes \& Ribeiro, 2019).

Em sequência, dois últimos problemas podem ser apontados sobre a literatura referente a gênero e segregação de gênero em cursos superiores no Brasil. Uma questão simples e surpreendentemente não discutida é que nenhum trabalho apresenta uma medida sintética sobre a intensidade da segregação. Isso é um problema porque, ao se analisar qualquer sistema heterogêneo, é possível que movimentos se compensem. Frequentemente, os estudos indicam o crescimento da participação feminina em um tipo de curso, mas simultaneamente também ocorrem movimentos de intensificação da segregação dos próprios cursos femininos ou masculinos. Como tudo isso se combina? Nenhum estudo brasileiro responde a essa pergunta.

Por fim, uma última questão não discutida na literatura brasileira relaciona-se com o nível de agregação adequado para conhecer o problema da segregação de gênero nas escolhas educacionais de ensino superior entre homens e mulheres no país. A mensuração das escolhas, frequentemente, é operacionalizada por meio de grupos de curso, mas tratada como cursos pelos trabalhos que utilizam dados dos censos demográficos. Em 2010, por exemplo, o IBGE seguiu o padrão da International Standard Classification of Education 1997 (Unesco, 1997) na mensuração dos cursos de ensino superior. 
Essa opção contém três níveis de agregação distintos, que variam das opções menos desagregadas para as mais desagregadas. A questão é que, quando se juntam cursos sob um mesmo rótulo, eles podem ter padrões distintos de segregação e esta pode acabar sendo subestimada, algo não mensurado por nenhum dos trabalhos supracitados. Assim, ao não serem esclarecidas as opções metodológicas de mensuração e ao não se apresentarem aos leitores os parâmetros empíricos que guiam as decisões, fica impossível discernir se as escolhas de agregação dos cursos são triviais ou não. No presente trabalho, estaremos atentos a essas consequências.

Infelizmente, no Brasil, os dados disponíveis não permitem que as hipóteses de pesquisa sejam colocadas nas escolhas em nível individual. Não é possível, por exemplo, entender os processos que produzem as escolhas de homens e mulheres no ensino superior - qual é sua sensibilidade às recompensas externas ou sua orientação aos futuros retornos financeiros no mercado de trabalho. Dessa forma, o avanço deste trabalho se dá no sentido de apresentar se há segregação das escolhas educacionais no Brasil, qual é o nível de agregação adequado para analisá-la, sua intensidade, onde ela se concentra e se existe alguma mudança em seu padrão temporal. Usamos, também, os dados mais adequados para monitorar essa questão: o Censo do Ensino Superior, que, surpreendentemente, é pouco acionado nas discussões sobre gênero e ensino superior no país e, sem dúvida, constitui a fonte de dados mais adequada para investigar essa questão, como argumentamos a seguir.

\section{Dados e Métodos}

\section{Dados}

A Pesquisa Nacional por Amostra de Domicílio (Pnad) é um levantamento realizado anualmente pelo IBGE nos domicílios brasileiros, a fim de apurar características gerais da população. Selecionamos as variáveis sexo, idade e escolaridade dos indivíduos para estudar a desigualdade de gênero no acesso ao nível superior. Essa base de dados fornece informações capazes de estimar as proporções de mulheres com ensino superior completo, em relação aos homens com o mesmo nível de educação em diferentes gerações. A partir desses dados, é possível ter uma noção da intensidade da estratificação vertical por gênero nas gerações passadas, o que permitirá contextualizar nosso problema de pesquisa.

Para estudar a segregação de gênero do ensino superior brasileiro, utilizamos os microdados do Censo da Educação Superior (CES) de 2002 e 2016, que contêm o número de matrículas masculinas e femininas, assim como os cursos em que esses registros estão alocados. O CES é uma base de dados organizada anualmente pelo Instituto Nacional de Estudos e Pesquisas Educacionais Anísio Teixeira (Inep) sobre as instituições de educação superior do país.

Os microdados possuem três variáveis: ano, matrículas por sexo e cursos (em quatro níveis de agregação diferentes, como explicaremos a seguir). Assim, é possível identificar se há ou não segregação em áreas específicas do sistema e como ela se comporta ao longo do tempo. No Apêndice on-line, ${ }^{1}$ encontram-se as variáveis e sua forma de codificação e as distribuições de quantidade de cursos (Tabela A1) e de matrículas por sexo (Tabela A2) para o período analisado.

A opção de produzir os indicadores a partir das matrículas se justifica porque o trabalho com outras fontes de informação relativas a ingressantes ou concluintes nos daria um retrato parcial da segregação de gênero no ensino superior brasileiro nos períodos avaliados. Se o trabalho se focasse somente nas e nos estudantes ingressantes no sistema, a distribuição da segregação de gênero de uma nova geração poderia ser confundida com a distribuição pregressa dos alunos, oferecendo um quadro limitado sobre nosso problema de pesquisa. O mesmo se aplicaria a concluintes, com a dificuldade 
adicional de confundir processos de segregação com processos de evasão e a seletividade de gênero que pode ocorrer dependendo do curso. Para contornar esses limites, optamos por trabalhar com matrículas, que são um indicador da distribuição de alunos em todos os pontos de suas trajetórias no sistema em um período.

A padronização dos microdados para construção de nossa base necessitou de diversos passos. O primeiro foi a exclusão de linhas do banco de dados de 2002 e 2016. Em sequência, para guiar a construção das bases de dados, juntamos as bases dos cursos presenciais, a distância e sequenciais de formação específica. Comparamos, para cada ano, os resultados da quantidade de cursos e do número de matrículas produzidos por nós e pelo Inep, em suas Sinopses Estatísticas e Instruções (Inep, 2003, 2017a, 2017b). As informações entre nossos cálculos e as sinopses não são consistentes.

Identificamos que, em 2002, a inconsistência entre os nossos dados e os da Sinopse do Censo do Ensino Superior 2002 (Inep, 2003) devia-se a dois fatores (não informados na documentação das bases) que precisam ser aplicados para os microdados relativos à educação presencial: exclusão de casos que não contêm informação sobre a data de início do funcionamento do curso; e exclusão de cursos de áreas básicas de ingresso (código 010B00 na variável do código de curso segundo o padrão da Organização para a Cooperação e Desenvolvimento Econômico - OCDE). No total, quatro cursos não continham informação sobre sua data de criação e quatro eram de áreas básicas de ingresso e não tinham a data de criação em 2002. Ou seja, para chegarmos aos 14.399 cursos reportados na Sinopse Estatística do Inep em 2002, eliminamos quatro linhas dos microdados. Desse modo, os cursos que não tinham matrículas registradas foram excluídos de nossos dados.

Para 2016, há 32.242 matrículas em cursos presenciais de bacharelado, licenciatura ou tecnológicos com "áreas básicas de ingresso", que apresentam código 010B00 na variável do código de curso segundo o padrão da OCDE. Seguindo a documentação (Inep, 2017a, p. 5), excluímos esses casos de nossa base. ${ }^{2}$ Entretanto, em sua própria Sinopse (Inep, 2017b), o Inep não faz a exclusão. Por exemplo, na aba 1.8 do arquivo o total de matrículas do ensino superior brasileiro é apresentado como 8.048.701, quando deveria ser 8.016.459, a partir da retirada das 32.242 matrículas em cursos de "áreas básicas de ingresso".

Um passo adicional necessário foi a exclusão de cursos que não possibilitavam uma comparação temporal, seja por aparecerem em apenas um dos dois anos avaliados ou por haver poucos cursos nos dois períodos (se um número igual ou menor que dez cursos existisse nos dos dois anos, o curso foi excluído de nossa análise). Seguindo esse critério, foram desconsiderados 37 cursos com um total de 4.669 matrículas em 2002 e 27 cursos com um total de 2.588 matrículas em $2016 .{ }^{3}$ Assim, nossa base de dados final conta com 14.956 cursos e 3.556 .037 matrículas, em 2002, e 34.410 cursos e 8.017.415 matrículas, em 2016.

Dois ajustes adicionais também guiaram nosso trabalho. $\mathrm{O}$ primeiro foi a recodificação de todos os cursos ligados às licenciaturas e à formação de professores. Os microdados do CES classificam os cursos segundo uma tipologia da OCDE. Nesse sentido, a classificação segue um padrão de codificação que permite o trabalho com quatro níveis de agregação, que vão dos cursos (seis dígitos de códigos, 1.162 categorias possíveis na tipologia) para áreas detalhadas de cursos (três dígitos de códigos, 90 categorias possíveis na tipologia), áreas específicas (dois dígitos, 23 categorias possíveis na tipologia) e áreas gerais (um dígito, nove opções na tipologia).

2 Mantivemos os cursos sequenciais de formação específica nos dois anos para garantir a comparabilidade temporal dos dados. Em 2002, esses cursos somavam 41.552 matrículas e, em 2016, 3.544 matrículas.

3 Os códigos e nomes dos cursos excluídos segundo o padrão OCDE (variável CO_OCDE e NO_OCDE nos microdados) são: 146F14 Formação de professor de economia doméstica; 215C02 Conservação e restauro de material cultural; 215F01 Fabricação de instrumentos musicais (não industrial); 347N01 Necessidades dos clientes; 420 C01 Ciências; $540 F 01$ Fabricação e processamento; 540F02 Produção joalheira; 620A01 Agricultura, engenharia florestal e pesca; $813 E 01$ Esportes; $813 F 02$ Formação de técnicos e treinadores esportivos; 813G02 Gestão desportiva e de lazer; 814 C01 Ciências domésticas; 814 E02 Economia doméstica; 860 S01 Serviços de segurança; 863C01 Ciência militar; e 863 F01 Formação militar. 
Nessa tipologia, qualquer curso de licenciatura é classificado como "formação de professor". Por exemplo, um curso de licenciatura em Matemática é classificado no código "145F18 Formação de professor de Matemática”. Como nossa pesquisa envolve entender como estudantes dos sexos feminino e masculino se ligam às áreas de estudo, essa sobreposição do grau (ex: licenciatura), em relação à área do conhecimento (ex: Matemática), não permite a produção dos resultados que desejamos, uma vez que a segregação de gênero se dá em áreas do conhecimento, não em tipos de grau. Assim, recodificamos todos os cursos de licenciatura de forma que a área se sobrepusesse ao grau. Em nosso exemplo, os cursos de licenciatura em Matemática foram recodificados para apenas “461M01 Matemática”. O resultado dessa opção é que o grupo de cursos ligados à área de licenciatura são estritamente aqueles relacionados à área de educação, como "142P01 Pedagogia", "144N01 Normal Superior", "142P03 Psicopedagogia" (todas nossas opções estão documentadas nas colunas que contêm o prefixo "nied" na aba "Códigos OCDE" do Apêndice on-line e os códigos de padronização dos dados estão disponíveis para qualquer pesquisador interessado). Por motivos que expomos a seguir, fizemos um reagrupamento de cursos e trabalhamos com um nível de detalhamento equivalente à opção de áreas específicas do conhecimento.

Além disso, os cursos dos dois outros graus possíveis - bacharelados e tecnológicos - já têm a codificação de seus nomes e grupos preponderantemente relacionada à área de especialização e dispensam a necessidade de seguir a mesma estratégia. Alertamos, assim, que grupos de cursos como "Eletricidade e Energia” contêm tanto cursos de bacharelado quanto tecnológicos agrupados nessa opção.

Duas outras opções também guiaram nossa decisão de lidar com cursos ligados às licenciaturas. Há um conjunto de cursos em que não é possível definir a área, como "145F02 Formação de professor de ciências", e que, portanto, não pode ser agrupado porque o termo "ciências" não é definido. Nessa lógica, não é possível entender se esses cursos deveriam ser agrupados com a Física, Biologia ou outra possibilidade; logo, tratamos esse conjunto como um grupo separado. Adicionalmente, ainda existem cursos ligados ao ensino técnico e profissionalizante, mas sem área de especialização definida. Estes também foram tratados como um grupo separado. ${ }^{4}$

Um passo adicional que julgamos necessário foi conduzir um reagrupamento de alguns cursos para diminuir a complexidade da apresentação dos resultados e deixar a classificação mais próxima da organização e dos nomes do ensino superior brasileiro. ${ }^{5}$

\section{Métodos}

O primeiro recurso metodológico que utilizamos para entender a segregação de gênero no ensino superior é a razão de chances (odds ratio), que permite comparar as chances de homens e mulheres com e sem ensino superior em diferentes faixas etárias na Pnad 2014, assim como as chances de estarem em diferentes cursos de ensino superior. Para os dados da Pnad, serão avaliadas pessoas que passaram pelo sistema educacional em diferentes pontos no tempo. Ao calcular a razão das chances masculinas e femininas em diferentes coortes, é possível ter uma ideia de quando as mulheres passaram a ter sobrerrepresentação nas matrículas no ensino superior brasileiro. A mesma estratégia será aplicada para entender como os cursos dentro do sistema se comportam, em função do sexo. O valor de referência para a análise da razão de chances é o seguinte: quanto mais próximo de 1, mais perto da igualdade ou paridade de gênero. Quando o foco se deslocar para a segregação de gênero dos cursos, serão invertidos os numeradores e separadas as análises para cursos com sobrerrepresentação feminina e masculina, com o intuito de deixar a análise mais intuitiva e comunicar diretamente quais são os cursos com maiores níveis de segregação entre os

\footnotetext{
4 Esses cursos são: 146F12 Formação de professor de disciplinas profissionalizantes do ensino médio; 146 F13 Formação de professor de disciplinas técnicas; 146F23 Formação de professor do ensino técnico; e 146P01 Licenciatura para a educação profissional e tecnológica.

5 Todas as nossas opções de recodificação estão documentadas na aba "Agregação" do arquivo disponível no link bit.ly/azulOUrosa.
} 
sexos. A razão de chances é uma medida insensível às margens da distribuição. A análise da evolução temporal do padrão de segregação de gênero no ensino superior brasileiro será feita comparando as razões de chances de cursos femininos e masculinos, em 2002 e 2016. Isso permitirá, portanto, identificar cursos que tiveram a segregação diminuída, aumentada ou estável.

O segundo recurso estatístico que mobilizamos para entender a segregação de gênero no ensino superior é o índice de associação (index of association) (Charles \& Grusky, 2004), representado na equação 1. Ele permite mensurar o grau em que as mulheres estão sub-representadas, em média, nos cursos, sem que o resultado seja afetado pelo tamanho destes ou pelo crescimento mais geral do sistema ao longo do tempo. Isso é relevante porque, em um contexto de intensa expansão educacional, o efeito do aumento do sistema tem de ser controlado para não afetar as conclusões sobre o que acontece com cada uma de suas partes. Nesse sentido, o índice é estratégico para fornecer uma medida sintética para a segregação e indicará qual é seu tipo de tendência: estabilidade, aumento ou redução.

$$
A=\exp \left(\frac{1}{J} * \sum_{j=1}^{J}\left\{\ln \left(\frac{F_{j k}}{M_{j k}}\right)-\left[\frac{1}{J} * \sum_{j=1}^{J} \ln \left(\frac{F_{j k}}{M_{j k}}\right)\right]\right\}^{2}\right)^{1 / 2}
$$

O índice de associação (A) foi criado para o estudo da segregação ocupacional. Nossa adaptação substitui ocupações por cursos. Em nosso contexto, $\mathrm{k}$ representa um ano, $\mathrm{j}$ corresponde a cursos e Fjk e Mjk são, respectivamente, o número de matrículas femininas e masculinas em u m curso em determinado ano. Charles e Grusky (1995) provam que A é invariante em transformações multiplicativas da razão de gênero e apontam que ele não é afetado por diferenças na estrutura ocupacional (no nosso caso, a estrutura do ensino superior no país). Assim, esse índice pode ser utilizado para comparar o grau de segregação das escolhas educacionais em dois pontos distantes no tempo. $\mathrm{O}$ índice mede o fator em que mulheres estão sub-representadas no curso médio e não é afetado pela proporção de estudantes em cursos ou grupos de cursos (Mann \& DiPrete, 2013). Se houvesse total equilíbrio na quantidade de homens e mulheres, o índice seria igual a 0 . Logo, qualquer número acima disso representa a presença de segregação e, quanto maior for, maior é a intensidade da segregação.

Por último, é importante destacar que calculamos o índice com diferentes agregações da variável de curso para verificar se opções mais ou menos agregadas afetam o nível de segregação observado. Como argumenta Barone (2011), ao não se atentar para o nível de agregação utilizado, os trabalhos podem suprimir a variação da segregação e subestimá-la. Por isso, apresentamos resultados para os dois anos (2002 e 2016) em três níveis de agregação diferentes, como detalhado a seguir.

\section{Resultados}

A primeira dimensão que analisamos é a desigualdade vertical, que se refere à distribuição dos indivíduos de diferentes sexos com ensino superior completo ou não. Para compreender as chances relativas de homens e mulheres de possuírem ensino superior em cada faixa etária, calculamos a razão de chances (odds ratio) para mulheres e homens com ensino superior completo em cada coorte de idade. Em primeiro lugar, para obter as chances de cada grupo individualmente, dividimos o total de homens e mulheres com ensino superior completo pelo número daqueles que não o tinham, para cada faixa etária analisada. Conforme sinalizado na Tabela 1 , depois de calculadas, as chances são mobilizadas na equação final de sua razão, indicando o nível de sobre ou sub-representação feminina no ensino superior em cada coorte analisada.

Se adicionarmos 24 anos em relação à data de nascimento dessas pessoas - uma referência de quando elas estariam se formando -, é possível identificar resultados convergentes com pesquisas 
anteriores (Beltrão \& Alves, 2009; Ribeiro \& Schlegel, 2015): a reversão da desigualdade de gênero para conclusão do ensino superior aconteceu na década de 1970 no Brasil. A única diferença entre o nosso trabalho e os dos autores é que somos capazes de avançar em gerações não observadas por eles e sinalizar que o hiato não apenas existe, mas é crescente e mais intenso nas gerações mais jovens.

Esse resultado aponta que a desigualdade de gênero pode ser entendida - do ponto de vista vertical (acesso a níveis de ensino mais elevados) - como menor escolarização média entre homens, ou seja, o grupo desprivilegiado é o de homens. Cabe, então, perguntar o porquê.

A literatura nacional observa que o fracasso escolar é mais acentuado entre os meninos, no que diz respeito tanto às taxas de repetência e evasão quanto à defasagem idade-série (Rosemberg, 2001; Pereira \& Carvalho, 2009). Segundo Carvalho (2001), diferentes dimensões da socialização escolar dos meninos produzem esse quadro de maior fracasso escolar: a própria sociabilidade de gênero na infância; as interações com os docentes e suas expectativas sobre os alunos, que se diferenciam entre meninos e meninas; e as expectativas familiares estabelecidas para cada gênero. Além disso, a literatura aponta que a desvantagem dos meninos no que diz respeito “à adaptação às normas, necessidade de autonomia e ao ofício de aluno dentro da escola acaba atrasando-os ou afastando-os do acesso à instituição, defasandoos em relação às meninas" (Pereira \& Carvalho, 2009, p. 691). Além disso, o trabalho é mais prejudicial ao percurso escolar dos meninos, ao passo que os afazeres domésticos operam de forma mais sutil para meninas (Artes \& Carvalho, 2010).

Rosemberg (2001) analisa o acesso à educação formal entre homens e mulheres e aponta que a discriminação feminina acontece de forma diferente, dependendo da fase da escolarização, além de transparecer mais na progressão das trajetórias escolares do que em barreiras específicas de acesso. Nesse sentido, o sistema escolar, mesmo tendo revertido a segregação em seu acesso, continua sendo horizontalmente excludente para as mulheres.

Em estudo realizado no Distrito Federal, Sousa (2017) aponta que há desigualdade na trajetória de meninos e meninas, sendo os meninos maioria entre os estudantes com trajetórias tidas como "desordenadas", que acabam sendo interrompidas. Conforme apontado nos resultados apresentados pela autora, para a coorte de alunos do $6^{\circ}$ ano do ensino fundamental em 2012, as meninas apresentavam $62 \%$ mais chances de estarem matriculadas no ensino médio do que os meninos em 2016 - período que levariam para progredir de uma série para outra. Independentemente da área considerada na rede de ensino do Distrito Federal, os meninos têm trajetórias menos eficientes do que as meninas, isto é, são um quantitativo menor em relação ao total de matriculados no ensino médio em 2016. Segundo a autora, "a eficiência nas trajetórias das escolas privadas da Ride-DF para ambos os sexos é maior em relação às escolas públicas e a diferença nessa eficiência entre meninos e meninas apresenta-se inferior ao constatado no sistema público de ensino" (Sousa, 2017, p. 67). Esse trabalho demonstra que acompanhar indivíduos é essencial, uma vez que as trajetórias escolares dos alunos são importantes para entender processos de evasão escolar. A evasão escolar masculina no ensino médio está correlacionada com maiores taxas de repetência no ensino fundamental (Leon \& Menezes-Filho, 2002). A pesquisa brasileira sobre esse tema se beneficiaria muito de dados longitudinais, que pudessem investigar esses diferentes processos e entender como eles estão associados às relações de gênero.

TABELA 1

RAZÕES DE CHANCES (MULHERES/HOMENS) POR COORTE DOS CONCLUINTES DO ENSINO SUPERIOR. BRASIL, 2014

\begin{tabular}{rccccccc}
\hline Coorte & $60-65$ & $55-59$ & $50-54$ & $45-49$ & $40-44$ & $35-39$ & $30-34$ \\
Razões de chance & 0,93 & 1,18 & 1,3 & 1,4 & 1,44 & 1,46 & 1,61 \\
\hline
\end{tabular}

Fonte: Elaboração das autoras com base em dados do IBGE, Pnad 2014. 
Feita a identificação do padrão de desigualdade vertical, partimos para a investigação da segunda característica relevante para a compreensão da relação entre gênero e escolhas educacionais: a estratificação horizontal. Essa noção diz respeito à distribuição de indivíduos dentro de um mesmo nível de ensino. No contexto da presente pesquisa, isso significa que iremos investigar como homens e mulheres se distribuem em diferentes grupos de cursos do ensino superior brasileiro.

Iniciamos nossa exploração de resultados com a mobilização do índice de associação (A) para entender a intensidade da segregação de gênero em dois anos diferentes. $\mathrm{O}$ índice representa o fator médio pelo qual mulheres ou homens estão sobrerrepresentadas(os) ou sub-representadas(os) em cada área. Utilizamos o índice para três tipos de agrupamento de curso (ver a classificação no Anexo indicado na nota número 1). Os agrupamentos seguem as três agregações presentes na estrutura da tipologia da OCDE, chamadas de área geral, área específica e área detalhada.

A diferença da intensidade da segregação captada pelo índice é passível de ser identificada ao movermos a análise do índice calculado da opção mais agregada para a mais desagregada. Essa diferença sinaliza que o nível de agregação dos cursos é algo relevante e, caso seja negligenciada, indutora de subestimação da segregação de gênero, tanto do ponto de vista estático (em um ponto no tempo) quanto do dinâmico (ao longo do tempo).

TABELA 2

ÍNDICE DE ASSOCIAÇÃO POR DIFERENTES AGREGAÇÕES DA VARIÁVEL DE CURSO. BRASIL, 2002 E 2016

\begin{tabular}{cccc}
\hline Ano & Área geral & Área específica & Área detalhada \\
2002 & 2,45 & 2,87 & 3,03 \\
\hline 2016 & 2,43 & 2,74 & 3,10 \\
\hline
\end{tabular}

Fonte: Elaboração dos autores com base em microdados do Censo do Ensino Superior (Inep).

Observando o índice calculado para as três agregações, fica evidente que o índice é sensível às opções de agrupamento. Nas duas versões mais agregadas, área geral, com nove categorias, e área especifica, com 22, os resultados apontam para uma redução da segregação de gênero no ensino superior brasileiro ao longo do tempo. Na versão mais desagregada, área detalhada, com 63 categorias, o contrário é identificado. Nessa opção, o resultado indica um aumento da segregação de gênero no ensino superior brasileiro. Isso mostra que opções agregadas subestimam a segregação, uma vez que cursos agrupados sob o mesmo rótulo têm padrões distintos de concentração de matrículas masculinas e femininas.

Dessa forma, optamos pela versão mais desagregada, que nos permite concluir que o nível mais adequado para descrever o padrão de segregação de gênero no ensino superior brasileiro é aquele que consegue captar a heterogeneidade da segregação de gênero das escolhas educacionais presente do sistema. Em relação à mudança temporal, não há nenhum sinal de diminuição do nível de segregação; pelo contrário, as escolhas de estudantes dos sexos masculino e feminino se mantiveram segregadas em um patamar parecido depois de 14 anos, com leve tendência de aumento.

A princípio, seria possível operar com uma versão ainda mais desagregada de classificação. Os microdados do CES dispõem de uma variável mensurada no nível do curso, conforme mencionado anteriormente, mas não é possível utilizá-la por duas razões. A primeira diz respeito ao que parecem ser mudanças na estratégia de mensuração das informações. Há cursos com muitas matrículas em 2002 e poucas em 2016. Como o sistema expandiu de uma forma geral e cursos em áreas de especialização parecidas (ex: Química e Bioquímica; Turismo e Hotelaria) tiveram flutuações de matrículas entre áreas de especialização próximas, isso pareceu ser um artefato da mensuração e não um processo empírico relevante. 
Felizmente, essas flutuações pouco afetam a mensuração de áreas. Por exemplo, em 2002, um curso pode ser classificado como Ciências Biológicas e, em 2016, como Biologia (ou o contrário). Em nossa estratégia de avaliação, a flutuação não afeta o resultado, pois os cursos são agrupados na opção Biologia e Bioquímica. Segundo, há também um critério de parcimônia. O trabalho com o nível mais desagregado de cursos envolveria a apresentação de 485 cursos para 2002 e 348 para 2016, o que não nos parece viável. Nossa escolha, então, resulta em 63 grupos de cursos, algo passível de análise, como esperamos demonstrar adiante.

Feita a descrição do nível de agregação adequado para a análise e a evolução temporal da segregação de gênero, é possível avaliar de forma desagregada como a segregação se comportou em cada grupo de cursos. Esse passo é necessário porque pode haver movimentos que se compensam, uma vez que determinados grupos de curso podem ter se dessegregado, ao mesmo tempo que outra área teve sua segregação intensificada. A área de Engenharia, por exemplo, que é sistematicamente mais masculina, pode ter tido uma inserção feminina significativa. Mas, se esse movimento ocorreu ao mesmo tempo que a área de Serviço Social, que é tradicionalmente mais feminina, teve ainda mais inserção de mulheres, as tendências se equilibrariam e não seriam refletidas em um índice agregado como o índice de associação. Para identificar e qualificar qual das duas tendências está ocorrendo, as áreas do conhecimento serão analisadas de forma desagregada. Para esse fim, voltaremos a mobilizar razões de chance.

TABELA 3

RAZÕES DE CHANCE NOS GRUPOS DE CURSO QUE TÊM SOBRERREPRESENTAÇÃO FEMININA EM 2002 E 2016. TABELA ORDENADA EM FORMA DECRESCENTE PARA VALORES DE 2016

\begin{tabular}{|c|c|c|c|}
\hline Grupos de curso & 2002 & 2016 & $\begin{array}{c}\text { Variação } \\
\text { 2016-2002 (\%) }\end{array}$ \\
\hline Serviços de Beleza & 5,7 & 30,5 & 438,0 \\
\hline Pedagogia e Formação de Professores & 9,3 & 10,2 & 10,0 \\
\hline Serviço Social e Orientação & 9,6 & 7,3 & $-24,0$ \\
\hline Secretariado e Trabalhos de Escritório & 5,6 & 5,9 & 6,0 \\
\hline Enfermagem e Atenção Primária & 4,5 & 4,2 & $-7,0$ \\
\hline Terapia e Reabilitação & 3,6 & 3,8 & 7,0 \\
\hline Psicologia & 4,8 & 3,3 & $-33,0$ \\
\hline Humanidades e Letras (cursos gerais) & 3,8 & 2,1 & $-46,0$ \\
\hline Farmácia & 1,5 & 2,0 & 33,0 \\
\hline Odontologia & 1,3 & 2,0 & 56,0 \\
\hline Biologia e Bioquímica & 1,7 & 1,9 & 11,0 \\
\hline Processamento de Alimentos & 1,8 & 1,8 & 1,0 \\
\hline Belas-Artes & 1,8 & 1,8 & 2,0 \\
\hline Línguas e Culturas Estrangeiras & 2,1 & 1,6 & $-24,0$ \\
\hline Biblioteconomia, Informação e Arquivos & 1,9 & 1,6 & $-16,0$ \\
\hline Viagens, Turismo e Lazer & 1,7 & 1,6 & $-9,0$ \\
\hline Arquitetura e Urbanismo & 1,3 & 1,5 & 12,0 \\
\hline Design e Estilismo & 1,1 & 1,3 & 20,0 \\
\hline Tecnologias de Diagnóstico e Tratamento & 1,1 & 1,2 & 4,0 \\
\hline Hotelaria, Restaurantes e Serviços & 1,7 & 1,1 & $-35,0$ \\
\hline Jornalismo e Reportagem & 1,1 & 1,1 & 2,0 \\
\hline Relações Internacionais & 1,2 & 1,1 & $-8,0$ \\
\hline Formação de Professor de Ciências & 1,3 & 1,0 & $-21,0$ \\
\hline Ciências Sociais & 1,2 & 1,0 & $-14,0$ \\
\hline
\end{tabular}

Fonte: Elaboração dos autores com base nos microdados do Censo do Ensino Superior 2002 e 2016 (Inep). 
TABELA 4

RAZÕES DE CHANCE NOS GRUPOS DE CURSO QUE TÊM SOBRERREPRESENTAÇÃO MASCULINA EM 2002 E 2016. TABELA ORDENADA EM FORMA DECRESCENTE PARA VALORES DE 2016

\begin{tabular}{|c|c|c|c|}
\hline Grupos de curso & 2002 & 2016 & Variação 2016-2002 \\
\hline Engenharia Mecânica e Metalurgia & 15,5 & 11,4 & $-26,0$ \\
\hline Eletrônica e Automação & 9,2 & 9,3 & 1,0 \\
\hline Ciência da Computação & 4,8 & 8,9 & 84,0 \\
\hline Eletricidade e Energia & 11,0 & 8,6 & $-21,0$ \\
\hline Proteção de Pessoas e de Propriedades & 3,8 & 8,5 & 122,0 \\
\hline Processamento da Informação & 3,8 & 8,2 & 115,0 \\
\hline Veículos a Motor, Construção Naval e Aérea & 11,9 & 6,8 & $-43,0$ \\
\hline Transportes e Serviços & 9,9 & 6,0 & $-39,0$ \\
\hline Uso do Computador & 4,3 & 5,8 & 36,0 \\
\hline Religião e Teologia & 2,8 & 4,1 & 48,0 \\
\hline Engenharia Civil e de Construção & 4,2 & 3,2 & $-23,0$ \\
\hline Física & 4,9 & 3,2 & $-35,0$ \\
\hline Materiais (madeira, papel, plástico, vidro) & 2,6 & 2,9 & 9,0 \\
\hline Engenharia e profissões correlatas & 4,6 & 2,4 & $-48,0$ \\
\hline Produção Agrícola e Pecuária & 3,1 & 2,3 & $-24,0$ \\
\hline Filosofia e Ética & 2,2 & 2,3 & 5,0 \\
\hline Ciências da Terra & 1,8 & 2,2 & 27,0 \\
\hline Economia & 1,9 & 2,2 & 16,0 \\
\hline Educação Física, Saúde e Saúde Pública* & 1,6 & 2,2 & 34,0 \\
\hline Mineração e Extração & 4,9 & 2,2 & $-55,0$ \\
\hline Estatística & 1,8 & 2,1 & 15,0 \\
\hline Matemática & 1,3 & 1,8 & 34,0 \\
\hline Música e Artes Cênicas & 1,3 & 1,7 & 31,0 \\
\hline Técnicas Audiovisuais e Produção de Mídia & 2,2 & 1,7 & $-24,0$ \\
\hline Proteção Ambiental & 2,4 & 1,7 & $-33,0$ \\
\hline Saúde e Segurança do Trabalho & 3,5 & 1,5 & $-56,0$ \\
\hline Engenharia Florestal e Silvicultura & 2,0 & 1,4 & $-32,0$ \\
\hline Marketing e Publicidade & 1,2 & 1,2 & $-5,0$ \\
\hline Química e Engenharia de Processos & 1,9 & 1,1 & $-42,0$ \\
\hline Administração e cursos correlatos & 1,6 & 1,1 & $-29,0$ \\
\hline Direito & 1,4 & 1,1 & $-25,0$ \\
\hline Química & 1,3 & 1,0 & $-22,0$ \\
\hline Ciências Ambientais & 1,5 & 1,0 & $-30,0$ \\
\hline
\end{tabular}

* O rótulo original é Saúde (áreas gerais). Renomeamos o grupo como “Educação Física, Saúde e Saúde Pública”.

Fonte: Elaboração dos autores com base nos microdados do Censo do Ensino Superior 2002 e 2016 (Inep).

Retomando o raciocínio da construção da estatística de razões de chances, quanto mais próxima de 1 for a razão, mais próximo da paridade de representação de gênero está aquele grupo de cursos. Uma razão de chances superior a 1 indica que o evento tem maior probabilidade de ocorrer no primeiro grupo (nesse caso com as mulheres na Tabela 3 e homens na Tabela 4). Os dados da Tabelas 3 e 4 foram obtidos ou com mulheres ou com homens no numerador, mostrando os cursos que têm sobrerrepresentação feminina ou masculina nos dois períodos avaliados.

Um primeiro resultado dessa análise desagregada emerge a partir da comparação dos cursos presentes em cada tabela. Observa-se que, dos 63 grupos de curso possíveis, 57 permaneceram predominantemente femininos ou masculinos entre 2002 e 2016. Os dados de ambas as tabelas 
mostram tendências de aumento e diminuição da segregação. Isso permite qualificar a interpretação do índice de associação: apesar de ele apontar uma virtual estabilidade da segregação no período avaliado, esse movimento não é estático, pois há cursos que aumentaram, diminuíram ou inverteram a segregação, mas esses movimentos se compensam.

Entre os cursos com maior presença feminina, 11 grupos diminuíram a segregação, enquanto, entre cursos masculinos, 18 tiveram a segregação reduzida. Isso indica que a atratividade de cursos com sobrerrepresentação feminina é menor para homens do que os cursos de sobrerrepresentação masculina para as mulheres.

A partir dos dados das Tabelas 3 e 4, podemos verificar se os eixos humanista-científico e cuidado-técnico são relevantes para a análise da segregação de gênero no ensino superior brasileiro. Aqui, temos um indicativo de que o padrão de segregação mais saliente é o cuidado-técnico e não o humanista-científico. Isso porque os cursos com sobrerrepresentação feminina são claramente ligados ao cuidado - Serviços de Beleza, Serviço Social e Orientação, Secretariado e Trabalhos de Escritório, Enfermagem e Atenção Primária -, enquanto os cursos com sobrerrepresentação masculina são aqueles com componente técnico - Engenharia Mecânica e Metalurgia, Eletrônica e Automação, Ciência da Computação, Eletricidade e Energia.

Apontamos, também, para as exceções ao padrão identificado. Os cursos nos grupos Farmácia; Biologia e Bioquímica; Processamento de Alimentos; e Tecnologias de Diagnóstico têm sobrerrepresentação feminina e o curso de Filosofia e Ética masculina. Não é o propósito dessa análise a avaliação individual de grupos de cursos, mas esses conjuntos apontam que, apesar de os padrões de segregação de gênero mais comuns em outros países - os eixos cuidado-técnico e humanista-científico - também ocorrerem no Brasil, isto não se dá de maneira uniforme.

Outro resultado de destaque é que dois grupos de curso, raramente citados nos estudos estrangeiros, são muito importantes no Brasil: Administração e cursos correlatos e Direito. Ambos apresentam uma virtual paridade de gênero em 2016. Como cursos dessas duas áreas concentravam $27 \%$ das matrículas, em 2002, e 26\%, em 2016, o que ocorre apenas nesses dois grupos de cursos ancora qualquer fenômeno relativo ao ensino superior brasileiro. Em qual tipo de perfil esses cursos se encaixam? Os cursos de Direito são ligados às ciências humanas e os da área de Administração são híbridos, uma vez que seus componentes curriculares envolvem conteúdos tanto da área de exatas (cálculos financeiros, por exemplo) como de humanas (gestão de pessoal). Nesse sentido, o ensino superior brasileiro foge do padrão internacional ao ter duas de suas áreas mais importantes com baixo nível de segregação em 2002 e menor ainda em 2016. O outro curso que mais concentra quantidade de matrículas em ambos anos, Pedagogia e Formação de Professores - com 10\% das matrículas em 2002 e 8\% em 2016 -, não segue a mesma tendência, sendo um dos grupos com maior sobrerrepresentação feminina e um dos indutores da segregação de gênero no ensino superior brasileiro.

TABELA 5

RAZÕES DE CHANCE NOS GRUPOS DE CURSO QUE INVERTERAM SUAS TENDÊNCIAS DE SEGREGAÇÃO ENTRE 2002 E 2016

\begin{tabular}{rrr} 
& Painel A - Cursos que inverteram a sobrerrepresentação na direção feminino-masculino & 2016 \\
\hline História e Arqueologia & 2002 & 0,72 \\
\hline & Painel B - Cursos que inverteram a sobrerrepresentação na direção masculino-feminino & 1,06 \\
\hline Contabilidade e Tributação & 0,71 & 1,66 \\
Veterinária & 0,73 & 1,49 \\
\hline Medicina & 0,86 & 1,01 \\
\hline
\end{tabular}

Fonte: Elaboração dos autores com base nos microdados do Censo da Educação Superior 2002 e 2016 (Inep). 
Por fim, analisamos os cursos que inverteram a segregação de gênero no período. Começamos por aqueles ligados ao grupo de História e Arqueologia. Como indicado no Painel A da Tabela 5, os cursos passaram de uma representação majoritariamente feminina para uma masculina. Somados a Filosofia e Ética, esses são os únicos cursos dá área das ciências humanas com sobrerrepresentação masculina. Em 2002, esses cursos tinham 1,06 matrícula feminina para cada matrícula masculina e, em 2016, passaram a ter 0,7. Essa é mais uma evidência contrária à saliência do eixo humanista-científico como padrão empiricamente proeminente para o entendimento da segregação de gênero nos cursos do ensino superior brasileiro.

As outras inversões ocorreram na direção oposta - grupos de curso predominantemente masculinos que se transformaram em predominantemente femininos ou equilibrados. Aqui, temos mais evidências de que cursos híbridos, ligados a negócios e administração, não se encaixam no padrão humanista-científico ou cuidado-técnico, como é o caso de Contabilidade e Tributação, e de que um grupo de cursos como o da área Têxtil, Roupas, Calçados e Couro (cursos de Confecção de Roupas, Engenharia de Produção Têxtil, Engenharia Têxtil, Indústria de Vestuário e Indústria Têxtil), eminentemente técnico, tem alto poder de atração entre estudantes do sexo feminino.

Finalmente, os cursos de Veterinária e Medicina são um sinal de que o eixo do cuidado pode ser um vetor indutor da participação feminina, que levou à sobrerrepresentação das mulheres, no caso da Veterinária, ou à paridade de sexo, na Medicina. Um tipo de análise interessante para pesquisadores conduzirem no futuro é entender como se dá a distribuição dos profissionais dessas áreas, na miríade de especializações que as compõem. Por exemplo, será que a inversão da segregação de gênero da área de Veterinária significa uma mudança na concentração profissional? Será que a área se deslocou de atividades masculinas (ex: manejo de gado) para mais femininas (ex: cuidado de pequenos animais)?

\section{Conclusão}

Os resultados que obtivemos e sua análise junto à literatura mobilizada lançam luz sobre como a segregação de gênero se configura no interior do sistema brasileiro de ensino superior. Em primeiro lugar, no que diz respeito à estratificação vertical de acesso, as mulheres levam vantagem em relação aos homens, pelo maior número de matrículas, desde a segunda metade da década de 1970. Essa não é propriamente uma novidade, mas chegamos a gerações mais recentes do que as anteriormente alcançadas por outras pesquisas e descobrimos que a desproporção da representação no ensino superior em favor das mulheres aumentou.

Esse resultado tem de ser interpretado à luz dos outros achados apresentados pela pesquisa. Os resultados da análise temporal do índice de associação indicam a estabilidade da segregação de gênero no ensino superior como um todo, mas exigem atenção à movimentação interna aos cursos em diferentes áreas, em que mudanças significativas ocorreram, inclusive de forma surpreendente, quando consideramos o esperado de acordo com a literatura internacional.

Esse é um passo importante para entender o quão significativa e resistente é a desigualdade horizontal. Se as mulheres se tornaram maioria no sistema de forma relativamente rápida, esse fato coexiste com a outra realidade: em 2016, ponto mais recente da análise, ainda há importante concentração de mulheres em determinadas áreas e pouca representação em outras. Conforme apontado por Charles e Bradley (2002), essa forma de segregação é mais resistente, uma vez que está sujeita às crenças de diferenças sexuais que permeiam a sociedade e influenciam no processo de escolha do campo de estudo. A partir dos dados do CES, observamos, em 2002, a clara configuração de campos femininos, caracterizados por grupos de curso principalmente ligados ao cuidado, e campos masculinos, majoritariamente ligados às áreas técnicas.

Ao contrário da literatura internacional, que tem destacado a estabilidade da segregação de gênero, os dados brasileiros se mostram heterogêneos em todas as direções. Há tanto cursos que reduziram a segregação, como os que aumentaram ou a inverteram.

Retomando os resultados, em 2016, observamos que as mulheres fizeram o caminho de inserção nos campos masculinos (na operacionalização deste estudo, Direito e Administração) de forma mais 
intensa do que os homens no sentido oposto. De acordo com England (2010), isso ocorre porque as mulheres recebem mais incentivos para adentrar áreas masculinas, sendo mais valorizadas do que seriam nas áreas femininas, enquanto os homens seriam desvalorizados adentrando áreas femininas, com perdas econômicas e culturais, o que implica a internalização de estereótipos de gênero e seu impacto na escolha de área de estudo (Charles \& Bradley, 2002; Barone, 2011).

Um passo importante para o futuro seria a elaboração de pesquisas longitudinais, que permitissem entender os mecanismos que estruturam os padrões agregados de segregação. As mulheres brasileiras escolhem as áreas de cuidado por rejeitarem uma hipotética discriminação em áreas dominadas por homens? Ou elas têm preferências por áreas com maior presença de contato interpessoal? E o contrário, qual o motivo de homens se concentrarem em determinadas áreas? Em relação a eles, é também necessário perguntar: por que homens têm carreiras educacionais menos longevas?

Adicionalmente, ainda alertamos que a redução da segregação ocupacional, por si só, não é suficiente para reduzir a desigualdade de gênero no mercado de trabalho. Diversas pesquisas (Galvão, 2015; Madalozzo \& Artes, 2017) apontam que, com as mesmas especializações, homens e mulheres têm retornos distintos no mercado de trabalho. Isso sinaliza que processos de autosseleção dentro das mesmas carreiras podem estar ocorrendo e concentrando mulheres nas especialidades com menores retornos (um exemplo hipotético na medicina seriam homens concentrados em oncologia e mulheres na pediatria). Além disso, carreiras masculinas e femininas têm diferentes trajetórias em organizações, o que também contribui para maiores e menores retornos. Por fim, não é possível, infelizmente, afastar a possibilidade da discriminação, velada ou não, que ainda afeta os ambientes de trabalho no país.

Ainda que não possa responder a essas perguntas, a presente pesquisa procurou contribuir para apresentar os números que confirmam a prevalência da segregação de gênero das escolhas educacionais no país, onde ela se concentra e como ela se comporta ao longo do tempo. Por meio desse conhecimento, é possível, no futuro, organizar ações que possibilitem que homens e mulheres estruturem suas decisões balanceando ações que reajam não somente aos estereótipos de gênero presentes nas famílias, escolas e sociedade, mas também a outros parâmetros. Espera-se, portanto, que estes possam promover resultados que conduzam, individualmente, a escolhas alinhadas a uma multiplicidade de parâmetros, para que, eventualmente, possam levar a uma distribuição de decisões mais competitivas e com retornos mais equilibrados entre homens e mulheres.

\section{Agradecimentos}

Esta pesquisa foi apoiada pela Fundação de Amparo à Pesquisa do Estado do Rio de Janeiro (Faperj) (contratos 010.002639/2019 e 010.001264/2016) e pelo Conselho Nacional de Desenvolvimento Tecnológico (CNPq) (contrato 400786/2016-8).

Agradecemos, em ordem alfabética, a Adriano Senkevics; Carlos Antônio Costa Ribeiro; pesquisadoras e pesquisadores do Grupo Estudos de Gênero, Educação e Cultura Sexual (EdGES-USP); Elisa Pereira Reis; Juliana de Castro Galvão; Maria Lígia Barbosa; e Natália Leão, por comentários a este texto.

\section{Referências}

Alves, J. E. D. (2003). Mulheres em movimento: Voto, educação e trabalho. REM.

Artes, A. C. A., \& Carvalho, M. P. de. (2010). O trabalho como fator determinante da defasagem escolar dos meninos no Brasil: Mito ou realidade? Cadernos Pagu, 34, 41-74. https://doi.org/10.1590/S010483332010000100004

Barone, C. (2011). Some things never change: Gender segregation in higher education across eight nations and three decades. Sociology of Education, 84(2), 157-176. https://doi. org/10.1177/0038040711402099 
Barone, C., \& Assirelli, G. (2020). Gender segregation in higher education: An empirical test of seven explanations. Higher Education, 79(1), 55-78. https://doi.org/10.1007/s10734-019-00396-2

Beltrão, K. I., \& Alves, J. E. D. (2009). A reversão do hiato de gênero na educação brasileira no século XX. Cadernos de Pesquisa, 39(136), 125-156. https://doi.org/10.1590/S0100-15742009000100007

Bobbitt-Zeher, D. (2007). The gender income gap and the role of education. Sociology of Education, 80(1), 1-22. https://doi.org/10.1177/003804070708000101

Brito, R. dos S. (2006). Intrincada trama de masculinidades e feminilidades: Fracasso escolar e meninos. Cadernos de Pesquisa, 36(127), 129-149. https://doi.org/10.1590/S0100-15742006000100006

Buchmann, C., DiPrete, T. A., \& McDaniel, A. (2008). Gender inequalities in education. Annual Review of Sociology, 34(1), 319-337. https://doi.org/10.1146/annurev.soc.34.040507.134719

Carvalho, M. P. D. (2001). Mau aluno, boa aluna? Como as professoras avaliam meninos e meninas. Revista Estudos Feministas, 9(2), 554-574. https://doi.org/10.1590/S0104-026X2001000200013

Carvalhaes, F., \& Ribeiro, C. A. C. (2019). Estratificação horizontal da educação superior no Brasil: Desigualdades de classe, gênero e raça em um contexto de expansão educacional. Tempo Social, 31(1), 195-233. https://doi.org/10.11606/0103-2070.ts.2019.135035

Cech, E. A. (2013). The self-expressive edge of occupational sex segregation. American Journal of Sociology, 119(3), 747-789. https://doi.org/10.1086/673969

Charles, M., \& Bradley, K. (2002). Equal but separate? A cross-national study of sex segregation in higher education. American Sociological Review, 67(4), 573-599. https://doi.org/10.2307/3088946

Charles, M., \& Bradley, K. (2009). Indulging our gendered selves? Sex segregation by field of study in 44 countries. American Journal of Sociology, 114(4), 924-976. https://doi.org/10.1086/595942

Charles, M., \& Grusky, D. B. (1995). Models for describing the underlying structure of sex segregation. American Journal of Sociology, 100(4), 931-971.

Charles, M., \& Grusky, D. B. (2004). Occupational ghettos: The worldwide segregation of women and men. Stanford University Press.

DiPrete, T. A., \& Buchmann, C. (2013). The rise of women: The growing gender gap in education and what it means for American schools. Russell Sage Foundation.

England, P. (2005). Emerging theories of care work. Annual Review of Sociology, 31(1), 381-399. https://doi. org/10.1146/annurev.soc.31.041304.122317

England, P. (2010). The gender revolution: Uneven and stalled. Gender and Society, 24(2), 149-166. https:// doi.org/10.1177/0891243210361475

England, P., \& Li, S. (2006). Desegregation stalled: The changing gender composition of college majors, 1971-2002. Gender \& Society, 20(5), 657-677. https://doi.org/10.1177/0891243206290753

Galvão, J. de C. (2015). O impacto da segregação de gênero nos cursos de graduação sobre o diferencial salarial entre homens e mulheres no Brasil [Dissertação de mestrado não publicada]. Universidade de Brasília.

Hirata, H. (2010). Teoria e práticas do care: Estado sucinto da arte, dados de pesquisa e pontos em debate. In N. Faria, \& R. Moreno (Orgs.), Cuidado, trabalho e autonomia. SOF.

Hirata, H., \& Guimarães, N. (2012). Cuidado e cuidadoras - As várias faces do trabalho do care. Editora Atlas S.A.

Instituto Nacional de Estudos e Pesquisas Educacionais Anísio Teixeira. (2003). Sinopse estatística da educação superior 2002.

Instituto Nacional de Estudos e Pesquisas Educacionais Anísio Teixeira. (2017a). Sinopse estatística da educação superior 2016.

Instituto Nacional de Estudos e Pesquisas Educacionais Anísio Teixeira. (2017b). Instruções para utilização dos microdados do Censo da Educação Superior.

Itaboraí, N. R. (2016). Trabalho feminino e mudanças nas famílias no Brasil (1976-2012): Uma perspectiva de classe e gênero. Revista Gênero, 16(2). https://doi.org/10.22409/rg.v16i2.31241

Leon, F. L. L. de., \& Menezes-Filho, N. A. (2002). Reprovação, avanço e evasão escolar no Brasil. Pesquisa e Planejamento Econômico, 32(3), 417-451. 
Madalozzo, R., \& Artes, R. (2017). Escolhas profissionais e impactos no diferencial salarial entre homens e mulheres. Cadernos de Pesquisa, 47(163), 202-221. https://doi.org/10.1590/198053143666

Mann, A., \& DiPrete, T. A. (2013). Trends in gender segregation in the choice of science and engineering majors. Social Science Research, 42(6), 1519-1541. https://doi.org/10.1016/j.ssresearch.2013.07.002

Ochsenfeld, F. (2016). Preferences, constraints, and the process of sex segregation in college majors: A choice analysis. Social Science Research, 56, 117-132. https://doi.org/10.1016/j.ssresearch.2015.12.008

Organização das Nações Unidas para a Educação, a Ciência e a Cultura. (1997). International Standard Classification of Education: ISCED 1997. Unesco Institute for Statistics.

Pereira, F., \& Carvalho, M. (2009). Meninos e meninas num projeto de recuperação paralela. Revista Brasileira de Estudos Pedagógicos, 90(226), 673-694. https://doi.org/10.24109/2176-6681.rbep.90i226.480

Ribeiro, C. A. C. (2007). Estrutura de classe e mobilidade social no Brasil. Edusc.

Ribeiro, C. A. C., \& Schlegel, R. (2015). Estratificação horizontal da educação superior no Brasil (1960 a 2010). In M. Arretche (Org.), Trajetórias das desigualdades. Como o Brasil mudou nos últimos cinquenta anos (p. 133-162). Editora Unesp.

Ricoldi, A., \& Artes, A. (2016). Mulheres no ensino superior brasileiro: Espaço garantido e novos desafios. Ex aequo - Revista da Associação Portuguesa de Estudos sobre as Mulheres, 33. https://doi.org/10.22355/ exaequo.2016.33.10

Rosemberg, F. (2001). Educação formal, mulher e gênero no Brasil contemporâneo. Revista Estudos Feministas, 9, 515-540. https://doi.org/10.1590/S0104-026X2001000200011

Schofer, E., \& Meyer, J. W. (2005). The worldwide expansion of higher education in the twentieth century. American Sociological Review, 70(6), 898-920. https://doi.org/10.1177/000312240507000602

Senkevics, A. S., \& Carvalho, M. P. de. (2015). Casa, rua, escola: Gênero e escolarização em setores populares urbanos. Cadernos de Pesquisa, 45(158), 944-968. https://doi.org/10.1590/198053143364

Sousa, A. P. de. (2017). Desigualdades nas trajetórias entre meninos e meninas nos anos finais do ensino fundamental da Ride-DF: Uma análise dos dados do censo escolar 2012-2016 [Dissertação de mestrado não publicada]. Universidade de Brasília.

Thébaud, S., \& Charles, M. (2018). Segregation, stereotypes, and STEM. Social Sciences, 7(7), 111. https://doi. org/10.3390/socsci7070111

Toledo, C. T., \& Carvalho, M. P. de. (2018). Masculinidades e desempenho escolar: A construção de hierarquias entre pares. Cadernos de Pesquisa, 48(169), 1002-1023. https://doi. org/10.1590/198053145496

Weeden, K. A., Gelbgiser, D., \& Morgan, S. L. (2020). Pipeline dreams: Occupational plans and gender differences in STEM major persistence and completion. Sociology of Education, 93(4), 297-314. https://doi.org/10.1177/0038040720928484

Nota sobre autoria

As autoras e autores participaram de todas as fases de elaboração e revisão do estudo.

\section{Disponibilidade de dados}

Os dados subjacentes ao texto da pesquisa estão informados no artigo.

\section{Como citar este artigo}

Mendes, T., Houzel, L., Milanski, B., Medeiros, C., Rocha, F. E., Elgaly, P., Almeida, V. de, \& Carvalhaes, F. (2021). Azul ou rosa? A segregação de gênero no ensino superior brasileiro, 2002-2016.

Cadernos de Pesquisa, 51, Artigo e07830. https://doi.org/10.1590/198053147830

Recebido em: 04 OUTUBRO 2020 | Aprovado para publicação em: 23 JULHO 2021 